\title{
Exchange Symmetries in Motzkin Path and Bargraph models of Copolymer Adsorption
}

\author{
E.J. Janse van Rensburg \\ A. Rechnitzer \\ Department of Mathematics and Statistics \\ York University, Ontario, Canada. \\ rensburg@mathstat.yorku.ca, andrew@mathstat.yorku.ca
}

Submitted: March 11, 2002; Accepted: April 23, 2002.

MR Subject Classifications: 05A15, 82B41

\begin{abstract}
In a previous work [26], by considering paths that are partially weighted, the generating function of Dyck paths was shown to possess a type of symmetry, called an exchange relation, derived from the exchange of a portion of the path between weighted and unweighted halves. This relation is particularly useful in solving for the generating functions of certain models of vertex-coloured Dyck paths; this is a directed model of copolymer adsorption, and in a particular case it is possible to find an asymptotic expression for the adsorption critical point of the model as a function of the colouring. In this paper we examine Motzkin path and partially directed walk models of the same adsorbing directed copolymer problem. These problems are an interesting generalisation of previous results since the colouring can be of either the edges, or the vertices, of the paths.

We are able to find asymptotic expressions for the adsorption critical point in the Motzkin path model for both edge and vertex colourings, and for the partially directed walk only for edge colourings. The vertex colouring problem in partially directed walks seems to be beyond the scope of the methods of this paper, and remains an open question. In both these cases we first find exchange relations for the generating functions, and use those to find the asymptotic expression for the adsorption critical point.
\end{abstract}

\section{Introduction}

Lattice models of adsorbing polymers have received significant attention in the physics literature over the last two decades $[8,13,14,29]$. These models are primarily based on the self-avoiding walk, a model which is known to pose formidable problems in combinatorics and probability theory [21]. Directed versions of lattice models of absorbing polymers 
are mathematically more tractable, while they also retain some of the rich combinatorial content so evident in more general models. The most well-known directed model of polymer adsorption is a model of Dyck paths $[15,16,23]$ and this model has also been considered in directed models of copolymer adsorption $[16,26]$.

Dyck paths are enumerated by the Catalan numbers and are connected to a myriad of other combinatorial objects; these are perhaps some of the most studied and best understood objects in combinatorics [27]. For example, an explicit solution for the generating function of Dyck paths enumerated according to their length and number of visits is known (see [15, 23] amongst many others); this is a directed model of adsorbing homopolymers. However, not all Dyck path problems have been solved; no similar explicit general expressions are known for coloured Dyck paths (these are models of adsorbing copolymers), except in the simplest of cases [16]. Further investigation of these models [26] suggests instead that a general solution would be unlikely, and only asymptotic expressions are known for critical points in these models. In particular, the asymptotic expression up to decaying terms for the critical point in a $\left\{A B^{p-1}\right\}^{*} A$-copolymer ${ }^{1}$ model of Dyck paths adsorbing in the main diagonal is

$$
a_{c}(p) \sim \frac{\sqrt{\pi}}{\zeta(3 / 2)} p^{3 / 2}+\frac{9 \sqrt{\pi} \zeta(5 / 2)}{8 \zeta(3 / 2)^{2}} p^{1 / 2}+1+O\left(p^{-1 / 2}\right)
$$

where only vertices coloured by $A$ are attracted by the main diagonal. It is also known that $a_{c}(1)=2$ [13], and that $a_{c}(2)=2+\sqrt{2}[16]$. This family of coloured Dyck paths is a directed model of a copolymer with two distinct comonomers arranged periodically. The majority of the polymer consists of a comonomer (represented by $B$-vertices) that does not interact with the adsorbing surface, while the periodic inhomogeneity (represented by $A$-vertices) are attracted onto the adsorbing surface. The length of the period of the colouring changes the behaviour of the system.

There are also other directed path models of polymer adsorption which could be studied. These include models of partially directed walks [23, 30], a special case of which is a model of bargraphs or histograms [24]. Alternatively, one may instead consider models of directed paths in other lattices. An example would be Motzkin paths [9], which is a model of (fully) directed paths on a triangular lattice, and confined to step only above the main diagonal. In both models of bargraphs and Motzkin paths, a two dimensional lattice model of an adsorbing polymer can be defined by letting the path be attracted to an adsorbing line. In models of Motzkin paths, the adsorbing line will be the main diagonal of the lattice, and it is possible for both vertices and edges to lie on this line, and so one may define two models in which vertices or edges interact with the adsorbing line. A similar situation is true for models of bargraphs, since both edges and vertices may lie in the adsorbing line (which is the $X$-axis). See figure 1 . This distinguishes these models from Dyck path models of directed polymer adsorption, where only vertices can be attracted into the main diagonal.

\footnotetext{
${ }^{1}$ In which the even numbered vertices are coloured periodically by repetitions of the block consisting of one $A$ followed by $p-1 B$ 's, and terminating in a single $A$.
} 

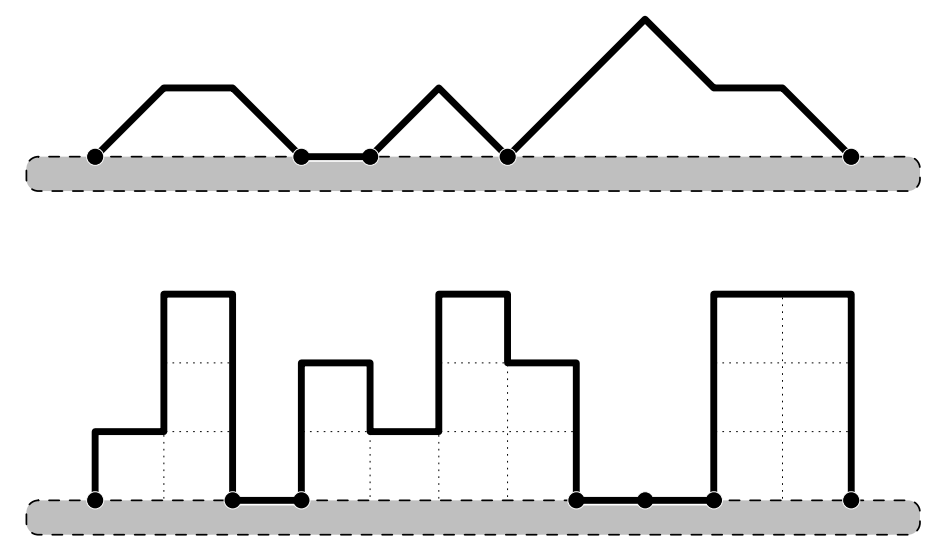

Figure 1: (top): A Motzkin path of length 11, with 1 edge-visit and 5 vertex-visits. (bottom): A bargraph of length 31, with 3 edge-visits and 7 vertex-visits.

In this paper we shall turn our attention first to models of adsorbing Motzkin paths, both with edges, and with vertices, interacting with the adsorbing line. This model can be turned into a model of copolymer adsorption by colouring the vertices with two colours, say $A$ and $B$, and where only colour $A$ will interact with the adsorbing line. The problem is unsolved for general colourings [26], and in this paper we only focus on the colouring $\left\{A B^{p-1}\right\}^{*} A$ with period $p$. Even in this case the model is unsolved - and we focus only on finding an asymptotic expression for the critical adsorption point in terms of $p$, similar to equation (1). The starting point is an exchange symmetry for Motzkin paths, analogous to the exchange symmetry for Dyck paths discussed in [26].

Models of adsorbing bargraphs are more difficult to analyse, and in this paper we only succeeded in solving a model where edges interact with the adsorbing line. We shall also briefly consider the model with vertices interacting with adsorbing line; while this model does exhibit an exchange-symmetry it is not as simple as that of Dyck paths or Motzkin paths and we have been unable to use it to find a solution.

A directed path in the square lattice (rotated by $45^{\circ}$ ) is a sequence of north-east and south-east steps. Such a path consists of edges and vertices, the first vertex is ordinarily placed on the origin, and the number of such paths with $n$ steps is $2^{n}$. A Dyck path is a directed path constrained to remain on or above the horizontal line $y=0$. The number of Dyck paths of $2 n$ edges is given by the Catalan numbers.

Motzkin paths are generalised Dyck paths which are able to step north-east, south-east and east. Like Dyck paths, Motzkin paths are constrained to remain on or above the line $y=0$. A vertex-visit in a Motzkin path is a vertex in the line $y=0$ which is also a vertex in the path. An edge-visit is an edge in the Motzkin path which is also an edge in the line $y=0$.

A second type of walk, called a partially directed walk, is a directed path in the square lattice that is only allowed to step north, south and east (while remaining self-avoiding). This means that a north step cannot be followed by a south step or vice-versa. If both the 
initial and final vertices of a partially directed walk are fixed in the line $y=0$, and the path is excluded from visiting vertices below the line $y=0$, then a bargraph is obtained (see Figure 1). Bargraphs are also models of adsorbing polymers: the $X$-axis is a natural adsorbing line and, much like Motzkin paths, both vertices or edges may be considered as visits in the adsorbing line.

The key object in this paper will be the generating function $G(z, v)$ of a generic model of directed or partially directed paths with $v$ the generating variable for vertex-visits in the adsorbing line (we shall use $w$ for edges-visits). In the thermodynamic sense, $v$ is an activity $^{2}$ conjugate to the number of visits in the model. By increasing the numerical value of the activity, paths with larger numbers of visits will contribute more to the generating function and determine the thermodynamic phase of the model. We introduce the generating variable $z$ conjugate to the length of the walks, and if $c_{n, k}$ is the number of paths of length $n$, with $k$ visits, then the generating function is given by:

$$
G(z, v)=\sum_{n \geq 0}\left(\sum_{k \geq 2} c_{n, k} v^{k}\right) z^{n}=\sum_{n \geq 0} Z_{n}(v) z^{n},
$$

where $Z_{n}(v)$ is the partition function of the model and it is related to the radius of convergence (and hence the growth constant) of $G(z, v)$ with respect to $z$ by

$$
z_{c}(v)=\lim _{n \rightarrow \infty}\left(Z_{n}(v)^{-1 / n}\right)=e^{-\mathcal{F}(v)},
$$

where $z_{c}(v)$ is the radius of convergence, and $\mathcal{F}(v)$ is the canonical limiting free-energy density [16]. It is worth noting that the derivative of the free energy (w.r.t. $\log (v)$ ) is the density of visits (or the energy density), and the second derivative of the limiting free energy is the specific heat which is a measure of the fluctations in the energy density. This relation between $z_{c}(v)$ and $\mathcal{F}(v)$ gives an explicit connection between the combinatorics and the thermodynamics of the model, and it is indeed possible to find the values of critical exponents associated with the adsorption transition from $z_{c}(v)$ [5].

Motzkin paths may be factored recursively into shorter Motzkin paths (see Figure 2), and consequently the generating function satisfies the following algebraic equation:

$$
M(z)=1+z M(z)+z M(z) z M(z)
$$

where $z$ is the generating variable for edges. Solving gives

$$
M(z)=[1-z-\sqrt{(1-3 z)(1+z)}] / 2 z^{2} .
$$

Motzkin paths are widely studied combinatorial objects; there is a bijection from these paths to the words in a one-coloured Motzkin algebraic language $[9,16]$. In our case, we will be interested in a modified version of the above. In particular, we shall introduce

\footnotetext{
${ }^{2}$ If we write $v=e^{\beta}$, then in the language of statistical mechanics $\beta$ can be called a fugacity, and $v$ is an activity. In these models the activity is a parameter which controls the strength of interaction of the paths with the wall.
} 
generating variables $v$ for vertex-visits and $w$ for edge-visits respectively, and reconsider the model. In these cases, we obtain generating functions $M(z, v)$ and $M(z, w)$ instead, and the key property of these will be their radii of convergence $z_{c}(v)$ and $z_{c}(w)$. In particular, these curves have a non-analytic point $v_{c}$ (and $w_{c}$ respectively) which corresponds to an adsorption transition in this model $[2,16,19,26]$. We are interested in the numerical values of these critical points; in particular how the position of the critical point depends upon the colouring of the path.

In Section 2 we first consider a Motzkin path model with vertex-visits. We show that the generating function of this model satisfies an exchange relation [26] which can be used to determine an asymptotic expression for the adsorption critical point $a_{c}(p)$ in a Motzkin path model of adsorbing directed copolymers whose vertices are coloured $\left\{A B^{p-1}\right\}^{*} A$ with $a$ the generating variable of $A$-vertex-visits:

$$
a_{c}(p)=\frac{2 \sqrt{3 \pi}}{9 \zeta(3 / 2)} p^{3 / 2}+\frac{13 \sqrt{3 \pi} \zeta(5 / 2)}{24 \zeta(3 / 2)^{2}} p^{1 / 2}+1+O\left(p^{-1 / 2}\right) .
$$

A similar analysis is done for an edge-coloured model with $A$-edge-visits weighted by $\alpha$; the dependence of the location of the critical point on the period of the colouring in that case is

$$
\alpha_{c}(p) \sim \frac{2 \sqrt{3 \pi}}{3 \zeta(3 / 2)} p^{3 / 2}+\frac{5 \zeta(5 / 2) \sqrt{3 \pi}}{8 \zeta(3 / 2)^{2}} p^{1 / 2}+1+O\left(p^{-1 / 2}\right) .
$$

Bargraphs are considered in Section 3. The basic quantity is $b_{n}$, the number of bargraphs of length $n$ steps, and the generating function associated with this model is

$$
B(z)=\left[1-z-z^{2}-z^{3}-\sqrt{\left(1-z^{4}\right)\left(1-2 z-z^{2}\right)}\right] / 2 z^{3} .
$$

More generally, we introduce generating variables $v$ for vertex-visits and $w$ for edge-visits to obtain the generating functions $B(z, v)$ and $B(z, w)$. In both these cases we may again colour the vertices or the edges by $\left\{A B^{p-1}\right\}^{*} A$ to obtain a bargraph model of adsorbing copolymers, with $a$ or $\alpha$ being the generating variables for vertex- $A$-visits and edge- $A$ visits respectively. In the case that edge- $A$-visits are considered, it is possible to find an asymptotic form for the location of the adsorption critical point with respect to $p$ :

$$
\alpha_{c}(p)=\frac{2 \sqrt{\pi} \sqrt{\sqrt{2}-1}}{\zeta(3 / 2)} p^{3 / 2}+\frac{3 \zeta(5 / 2) \sqrt{\pi} \sqrt{58 \sqrt{2}-2}}{\zeta(3 / 2)^{2}} p^{1 / 2}+1+O\left(p^{-1 / 2}\right) .
$$

but the model with vertex- $A$-visits remains seemingly intractable; our methods seem not able to allow the determination of an asymptotic expression for $a_{c}(p)$. The case of edge$A$-visits are considered fully in Section 3.2 with its asymptotic analysis in Section 3.4. We also indicate in Section 3.3 why the case of vertex- $A$-visits is not treatable by the techniques in this paper.

\section{Adsorbing Motzkin Paths}

We first review a model of adsorbing Motzkin paths with vertex-visits. The most fundamental quantity in this model is $m_{n, l}$, the number of Motzkin paths with $n$ steps and 
$l$ vertex-visits. The two variable generating function is $M(z, v)=\sum_{n=0}^{\infty} \sum_{l=0}^{n} m_{n, l} l^{l} z^{n}$, and we show how it may be derived in Section 2.1. The edge-visit model is examined in Section 2.2. In both these models we show that the two variable generating function satisfies an exchange relation, which shall be useful in analysing models of coloured adsorbing Motzkin paths.

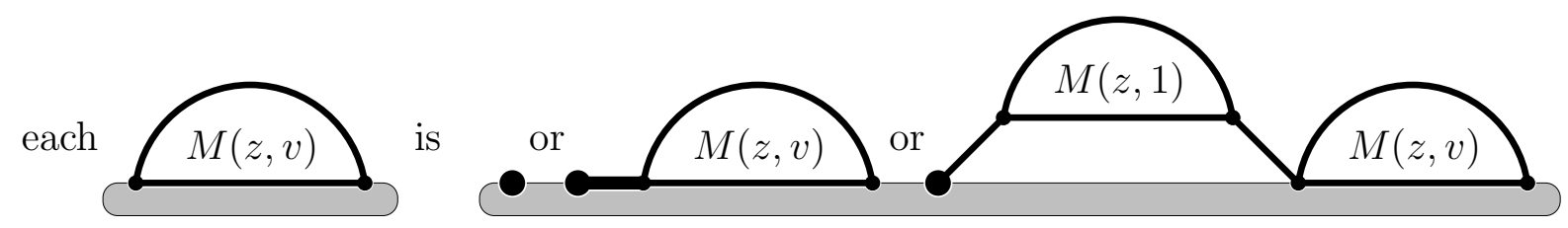

Figure 2: The canonical factorisation of Motzkin paths.

\subsection{Motzkin Paths with Vertex-visits}

Motzkin paths may be factored recursively in terms of shorter Motzkin paths. In particular, every adsorbing Motzkin path is either a single vertex or is a horizontal edge followed by another adsorbing Motzkin path, or may be factored into a north-east edge, a Motzkin path, a south-east edge (terminating on the axis) and then an adsorbing Motzkin path - this is illustrated in Figure 2. The factorisation in Figure 2 may be translated into the following algebraic equation satisfied by the generating function:

$$
M(z, v)=v+v z M(z, v)+v z^{2} M(z, 1) M(z, v) .
$$

Solving first for $M(z, 1)$ gives equation (5), and then equation (10) can be used to find $M(z, v)$ :

$$
M(z, v)=\frac{v}{1-v z-v z^{2} M(z, 1)}
$$

The radius of convergence of $M(z, v)$ can be found by examining the function's singularities. There is a line of square root branch points along $z=1 / 3$ in the $v z$-plane, and a curve of simple poles along $z=[1-v+\sqrt{(v+3)(v-1)}] / 2 v$. The limiting free energy $\mathcal{F}(z)$ in equation $(3)$ is determined by the radius of convergence, and there is exactly one non-analytic point in it at $v_{c}=3 / 2$. Further examination of the model shows that this is the adsorption critical point. The critical curve is a plot of the radius of convergence, and is given by

$$
z_{c}(v)=\left\{\begin{array}{cl}
1 / 3, & w \leq 3 / 2 ; \\
\frac{1-v+\sqrt{(v+3)(v-1)}}{2 v}, & w>3 / 2 ;
\end{array},\right.
$$

An exchange relation which is satisfied by $M(z, v)$ may be found using the approach described in Figure 3. This relation is very similar to the exchange relation found for Dyck paths in [26]: 
Theorem 1. Motzkin paths with vertex-visits weighted by $v$ satisfies the exchange relation

$$
v M(z, v)(M(z, 1)-1)=(M(z, v)-v) M(z, 1) .
$$

Solving this relation gives:

$$
M(z, v)=\frac{v M(z, 1)}{v+(1-v) M(z, 1)} .
$$

Proof. Consider a Motzkin path consisting of more than one vertex, in which no visitvertices are (yet) weighted. Then, starting from the left and working towards the right weigh the visit vertices by $v$, stopping somewhere before the last vertex of the path is reached. This path is the union of a Motzkin path (in which all the visits are weighted by $v$ ) and an unweighted Motzkin path (in which the visits are not weighted). The situation is depicted in the top half of Figure 3.

If we now weight the next vertex visit, then the situation is now depicted by the bottom half of Figure 3. Further, since the unweighted path becomes shorter and the weighted path longer, the initial unweighted path and the final weighted path must both consist of more than a single vertex. Summing over all possible conformations gives equation (13). We note that " -1 " and " $-v$ " are present in the equation due to the condition on the lengths of the initial unweighted and the final weighted Motzkin paths.

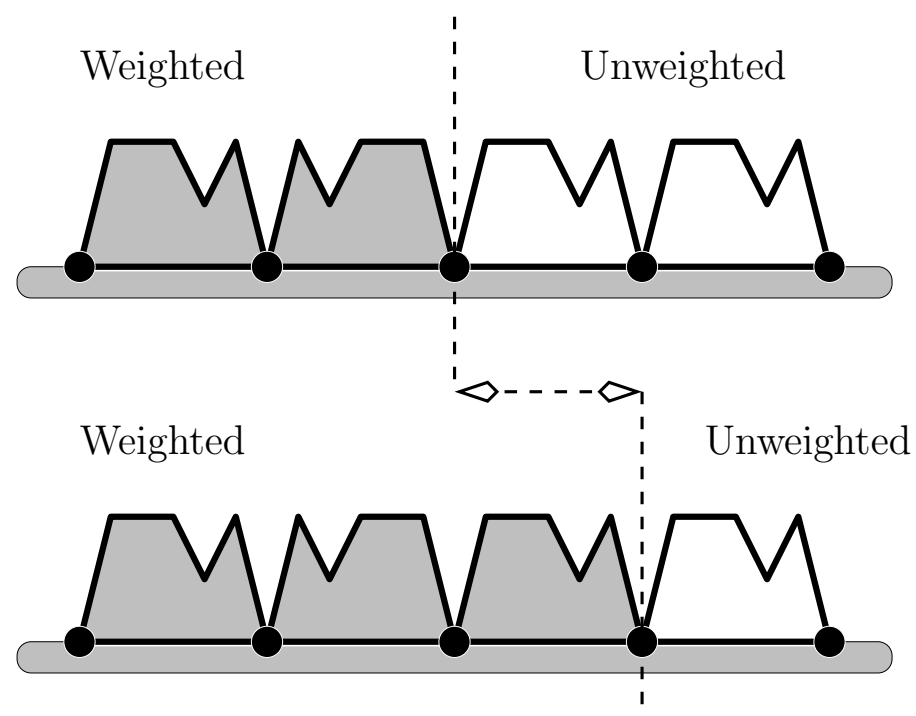

Figure 3: The vertex-visit exchange relation. The top diagram shows a (possibly empty) weighted Motzkin path attached to a non-empty unweighted path. By weighting the next vertex-visit one arrives at the bottom diagram which shows a non-empty weighted path attached to a (possibly empty) unweighted path.

This exchange relation may be generalised to a Motzkin path model of $\left\{A B^{p-1}\right\}^{*} A$ copolymer adsorption, using arguments similar to those in a Dyck path model [26]. Consider a Motzkin path of length $0 \bmod p$ and colour its vertices from left to right by 
$\left\{A B^{p-1}\right\}^{*} A$, and let $v a$ generate vertex-visits of colour $A$ and let $v$ generate vertex-visits of colour $B$.

Definition 1. Fix the period of the colouring $\left\{A B^{p-1}\right\}^{*} A$ to be $p$. We define $M(z, v, a \mid p)$ to be the generating function of this model of Motzkin paths of length 0 mod $p$ with vertices labelled by $\left\{A B^{p-1}\right\}^{*} A$, with all $B$-vertex-visits generated by $v$ and $A$-vertex-visits generated by va.

Theorem 2. The generating function $M(z, v, a \mid p)$ satisfies the following exchange relation:

$$
a M(z, v, a \mid p)(M(z, v, 1 \mid p)-v)=(M(z, v, a \mid p)-v a) M(z, v, 1 \mid p) .
$$

Solving for $M(z, v, a \mid p)$ then gives

$$
M(z, v, a \mid p)=\frac{v a M(z, v, 1 \mid p)}{v a+(1-a) M(z, v, 1 \mid p)} .
$$

Proof. Consider Figure 3 again. The top picture consists of a coloured part with $B$-vertexvisits weighted by $v$ and $A$-vertex-visits weighted by $v a$ (and this path may be empty), followed by an uncoloured path with all vertex-visits weighted by $v$ (this path is not empty). Thus, conformations of this type are generated by $M(z, v, a \mid p)(M(z, v, 1 \mid p)-v))$. Now, starting at the last vertex in the coloured path (which is always an $A$-vertexvisit) and continue to colour the path until the next $A$-vertex-visit is reached. This visit will now contribute weight $v a$ to the generating function, instead of $v$. Such a visit always exists since the path has length $0 \bmod p$, and the second part of the path is nonempty. In this case, the bottom picture in Figure 3 is obtained, and it is generated by $(M(z, v, a \mid p)-v a) M(z, v, 1 \mid p)$ (since the first part of the path that may not be empty). Finally, notice that the only difference between the two paths in the top and bottom of Figure 3 is a factor of $a$ introduced to account for the colour of the new $A$-vertex-visit. Thus, the identity follows.

If $v=1$, then a Motzkin path model coloured by $\left\{A B^{p-1}\right\}^{*} A$ with only the $A$-vertexvisits weighted by $a$ is obtained. A second interesting model is obtained if one first puts $a \rightarrow 1 / a$ and then $v=a$ : This gives a model of Motzkin paths coloured by $\left\{B A^{p-1}\right\}^{*} B$ with $A$-vertex-visits weighted by $a$.

Observe that the full generating function of all Motzkin paths (of any length) coloured by $\left\{A B^{p-1}\right\}^{*} A$ cannot be obtained from Theorem 2 . On the other hand, this generating function is known for a Dyck path version of this model [26], and following similar arguments, one may in fact write down the full generating function for Motzkin paths. Define $F(z, v, a \mid p)$ to be the full generating function of all Motzkin paths (of any length) whose vertices are coloured by $\left\{A B^{p-1}\right\}^{*} A$. Then $F(z, v, a \mid p)$ may be found as follows.

Theorem 3. Let $\bar{F}(z, v \mid p)$ be the generating function of the subset of Motzkin paths counted by $F(z, v, 1 \mid p)$, but with exactly one A-vertex-visit (their first vertices). Then

$$
F(z, v, a \mid p)=M(z, v, a \mid p) \bar{F}(z, v \mid p) / v
$$


from which follows

$$
\bar{F}(z, v \mid p)=v F(z, v, 1 \mid p) / M(z, v, 1 \mid p) .
$$

Thus, the full generating function is given by the following expression:

$$
F(z, v, a, \mid p)=\frac{v a F(z, v, 1 \mid p)}{v a+(1-a) M(z, v, 1 \mid p)}
$$

Proof. Cutting any path at its last $A$-visit factors it into a vertex-coloured path of length $0 \bmod p$ and a path of arbitrary length that contains only a single $A$-visit being its first vertex. Setting $a=1$ in this equation then gives the expression for $\bar{F}$. Substituting the result of the previous theorem gives the final expression.

\subsection{Motzkin Paths with Edge-visits}

An alternative Motzkin path model of polymer adsorption is obtained if one considers the adsorption of edges (rather than vertices) onto the adsorbing axis. The generating function as $M(z, w)$, where $w$ is conjugate to the number of edge-visits may be found using similar arguments to the vertex-visits case discussed in the last section. The factorisation in Figure 2 can be used to write down a functional relation for $M(z, w)$ :

$$
M(z, w)=1+w z M(z, w)+z^{2} M(z, 1) M(z, w),
$$

and one may solve explicitly for $M(z, 1)$ to obtain equation $(5)$, and then solve again for $M(z, w)$ : The result is

$$
M(z, w)=\frac{2}{1+z-2 w z+\sqrt{(1+z)(1-3 z)}} .
$$

$M(z, w)$ has a line of square root branch points along $z=1 / 3$ in the $w z$-plane, and a curve of simple poles along $z=(w-1) /\left(w^{2}-w+1\right)$; these singularities determine the radius of convergence of $M(z, w)$ : which is

$$
z_{c}(w)=\left\{\begin{array}{cl}
1 / 3 & w \leq 2 \\
\frac{w-1}{w^{2}-w+1} & w>2
\end{array}\right.
$$

The limiting free energy is $\mathcal{F}(w)=-\log z_{c}(w)$ [16] and this determines the thermodynamic properties of this model. There is a non-analytic point in $\mathcal{F}(w)$ at the intersection of the line of branch points $z=1 / 3$ with the curve of poles at $w_{c}=2$.

$M(z, w)$ also satisfies an exchange relation, but its form is somewhat more complicated than the exchange relation for $M(z, v)$ in Theorem 2. More careful arguments are also needed to find it. Let $H(z, w)$ be the generating function of all Motzkin paths with first edge horizontally in the adsorbing line. Paths counted by $H(z, w)$ can be obtained from $M(z, w)$ by appending a single horizontal edge on the leading vertex of every path; this shows that

$$
H(z, w)=w z M(z, w)
$$




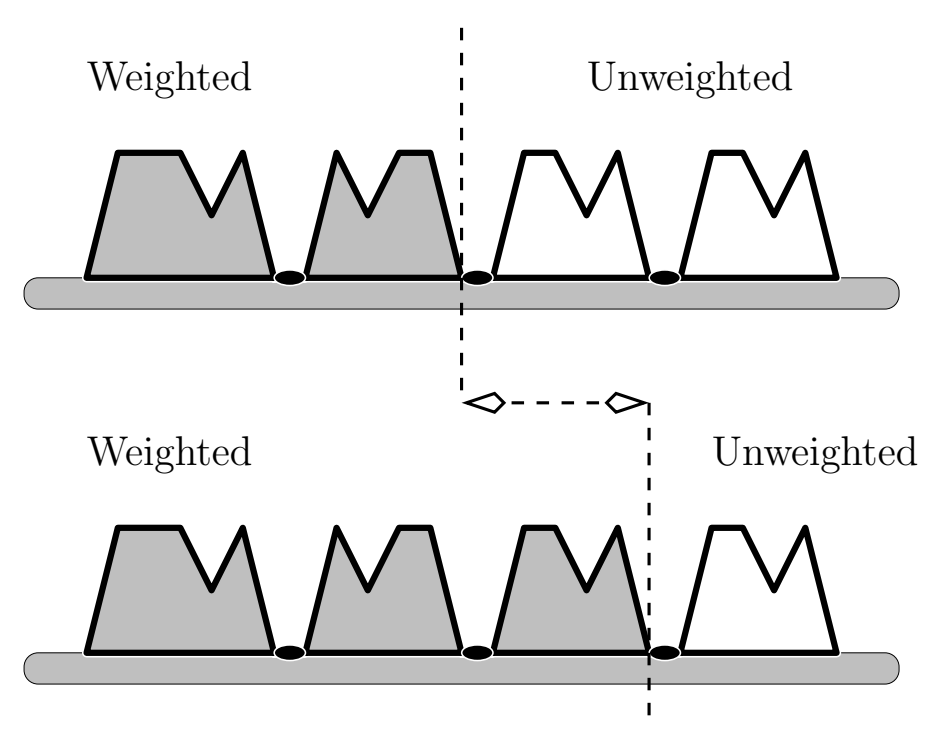

Figure 4: Edge-visit exchange relation.

Motzkin paths counted by $H(z, w)$ are called anchored. The important observation is that every Motzkin path with at least one edge-visit can be decomposed into a Motzkin path, and an anchored Motzkin path. Together with the techniques developed in reference [26] this observation gives the following theorem.

Theorem 4. Motzkin paths with edge-visits weighted by $w$ satisfies the exchange relation

$$
w M(z, w) H(z, 1)=(M(z, w)-M(z, 0))(H(z, 1)+1) .
$$

Solving for $M(z, w)$ from this exchange relation then shows that

$$
M(z, w)=\frac{M(z, 0)(1+H(z, 1))}{1+(1-w) H(z, 1)}=\frac{M(z, 1)}{1+(1-w) H(z, 1)} .
$$

Proof. The exchange relation is found by first considering a partially weighted Motzkin path, as in Figure 4. The path consists first of a weighted Motzkin path, followed by a non-empty, but unweighted anchored Motzkin path (generated by $H(z, 1)$ ). These partially weighted paths are then generated by $M(z, w) H(z, 1)$. If the next edge-visit is assigned the weight $w$, then the walks consists first of a weighted Motzkin path with at least one edge-visit which is counted by $M(z, w)-M(z, 0)$, followed by the empty path or an unweighted anchored Motzkin path; all generated by $1+H(z, 1)$. In other words, $w M(z, w) H(z, 1)=(M(z, w)-M(z, 0))(1+H(z, 1))$.

The generating function $M(z, 0)$ may be replaced in equation (14) by setting $w=1$ in the exchange relation which gives $M(z, 1)=M(z, 0)(1+H(z, 1))$, or by noting the more general relation $M(z, w)=M(z, 0)(1+H(z, w))$. Making this substitution completes the proof. 
As was the case for Dyck paths and the previous Motzkin path model, we see that the exchange relation (24) is not particularly useful for the homopolymer case, but it is very useful when applied to Motzkin paths of length $0 \bmod p$ with edges coloured in sequence by $\left\{A B^{p-1}\right\}^{*}$. We define the following generating variables in this model: $z$ will generate edges; $w$ generates edge-visits, whether coloured $A$ or $B$, and $\alpha$ generates only $A$-edgevisits. As before, we shall also be interested in Motzkin paths with first edge horizontally in the adsorbing line; these will again be called anchored Motzkin paths.

Define the following generating functions of Motzkin paths:

Definition 2. Fix the period of the colouring $\left\{A B^{p-1}\right\}^{*}$ to be $p$. Define the following:

- $M(z, w, \alpha \mid p)$ is the generating function of Motzkin paths of length $0 \bmod p$ with edges labeled by $\left\{A B^{p-1}\right\}^{*}$ and wa generates $A$-edge-visits and $w$ generates $B$-edgevisits;

- $H(z, w, \alpha \mid p)$ is the generating function of all anchored Motzkin paths of length 0 $\bmod p$ with edges labeled by $\left\{A B^{p-1}\right\}^{*}$ and $w \alpha$ generates $A$-edge-visits and $w$ generates B-edge-visits;

We first find an exchange relation for $H(z, w, \alpha \mid p)$ and $M(z, w, \alpha \mid p)$, using arguments similar to those in Theorem 4 above. All edge-visits are weighted by $w$, but we shall now proceed by weighing $A$-edge-visits with $\alpha$.

Theorem 5. The generating function $M(z, w, \alpha \mid p)$ satisfies the exchange relation

$$
\alpha M(z, w, \alpha \mid p) H(z, w, 1 \mid p)=(M(z, w, \alpha \mid p)-M(z, w, 0 \mid p))(1+H(z, w, 1 \mid p)) .
$$

It follows that

$$
M(z, w, 1 \mid p)=M(z, w, 0 \mid p)(1+H(z, w, 1 \mid p))
$$

and so

$$
M(z, w, \alpha \mid p)=\frac{M(z, w, 1 \mid p)}{1+(1-\alpha) H(z, w, 1 \mid p)}
$$

Proof. The argument is similar to the proof of Theorem 5. Consider again Figure 4 and observe that the top conformation consists of a coloured Motzkin path generated by $M(z, w, \alpha \mid p)$ followed by an anchored Motzkin path with edge-visits weighted by $w$, but with all $A$-edge-visits weighted by $w$ (so that $\alpha=1$, and these are generated by $H(z, w, 1 \mid p))$. In other words, these conformations are generated by $M(z, w, \alpha \mid p) H(z, w, 1 \mid p)$. Find the next $A$-edge-visit in these conformations, and weight it with an extra factor $\alpha$. The result is a fully weighted coloured Motzkin path with at least one $A$-edge-visit, generated by $M(z, w, \alpha \mid p)-M(z, w, 0 \mid p)$; followed by either the empty path or an anchored Motzkin path, together generated by $1+H(z, w, 1 \mid p)$. It is important to note that this construction leaves all paths with length $0 \bmod p$, consistent with the restriction on path-length in this model. The result is the claimed identity. 
If $w=1$, then a model of $\left\{A B^{p-1}\right\}^{*}$ coloured Motzkin paths are obtained, with the $A$-edge-visits weighted by $\alpha$. Other models can also be found; if we first let $\alpha \rightarrow 1 / \alpha$, followed by $w=\alpha$, then a $\left\{B A^{p-1}\right\}^{*}$ coloured Motzkin path is obtained, with only the $A$-edge-visits weighted by $\alpha$.

Observe also that $M(z, w, \alpha \mid p)$ is the generating function of only those Motzkin paths of length $0 \bmod p$, and coloured by $\left\{A B^{p-1}\right\}^{*}$. From a combinatorial point of view, one is really interested in the full generating function of this model, $G(z, w, \alpha \mid p)$. In the case of vertex-visits discussed in the Section 2.1 we were able to find the full generating function $F(z, w, a \mid p)$, but in the edge-visit model we are unable to solve for $G(z, w, \alpha \mid p)$ in terms of $M(z, w, \alpha \mid p)$. On the other hand, from the physical point of view we are more interested in the location of a non-analytic point in the radius of convergence $z_{c}(w, \alpha \mid p)$ of $G(z, w, \alpha \mid p)$. We can show that $z_{c}(w, \alpha \mid p)$ is also the radius of convergence of $M(z, w, \alpha \mid p)$, and so information about $G(z, w, \alpha \mid p)$ can be obtained by studying $M(z, w, \alpha \mid p)$ instead.

Theorem 6. The radius of convergence of the generating functions $G(z, w, \alpha \mid p)$ and $M(z, w, \alpha \mid p)$ are both given by $z_{c}(w, \alpha \mid p)$.

Proof. Suppose that $z_{c}(w, \alpha \mid p)$ is the radius of convergence of $G(z, w, \alpha \mid p)$. Suppose that

$$
G(z, w, \alpha \mid p)=\sum_{n=0}^{\infty} Z_{n}(w, \alpha \mid p) z^{n}
$$

where $Z_{n}(w, \alpha \mid p)$ is the canonical partition function of Motzkin paths coloured in sequence by $\left\{A B^{p-1}\right\}^{*}$ and of arbitrary length $n$. We remind the reader (see equation (3)) that

$$
z_{c}(w, \alpha \mid p)=\lim _{n \rightarrow \infty}\left(Z_{n}(w, \alpha \mid p)\right)^{-1 / n}
$$

and so the radius of convergence of $M(z, w, \alpha \mid p)$ is given by taking this last limit along a subsequence $\{p, 2 p, 3 p, \ldots, m p, \ldots\}$, and so all we need to prove is that the limit exists. We do this by demonstrating that $Z_{n}$ satisfies a super-multiplicative relation. This implies the existence of the limit (see [31]) and so will complete the proof.

Let

$$
Z_{n}(w, \alpha \mid p)=\sum_{V, U} m_{n}(V, U) w^{V} \alpha^{U}
$$

where $m_{n}(V, U)$ is the number of Motzkin paths coloured in sequence by $\left\{A B^{p-1}\right\}^{*}$ and of length $n$ with $V$ edge-visits of which $U$ are $A$-edge-visits. Fix integers $n_{1}, V_{1}, U_{1}$. Paths counted by $m_{n_{1}}\left(V_{1}, U_{1}\right)$ can be concatenated with paths counted by $m_{n_{2}}\left(V-V_{1}, U-U_{1}\right)$ as follows: Let $q$ be the smallest integer so that $n_{1} \leq q p$, and append $B$-edge-visits after the last vertex of the paths counted by $m_{n_{1}}\left(V_{1}, U_{1}\right)$ until paths of length $q p$ are obtained with $V_{1}+q p-n_{1} B$-edge-visits. Since the number of edges in these paths is a multiple of $p$, we can concatenate paths counted by $m_{n_{2}}\left(V-V_{1}, U-U_{1}\right)$ to the last vertex to obtain new paths of length $q p+n_{2}$ consistently coloured by $\left\{A B^{p-1}\right\}^{*}$. These paths have length 
$q p+n_{2}, V+q p-n_{1}$ with $B$-edge-visits and $U$ edge-visits of colour $A$. Summing over $V_{1}$ and $U_{1}$ shows that

$$
\sum_{V_{1}=0}^{V} \sum_{U_{1}=0}^{U} m_{n_{1}}\left(V_{1}, U_{1}\right) m_{n_{2}}\left(V-V_{1}, U-U_{1}\right) \leq m_{q p+n_{2}}\left(V+q p-n_{1}, U\right)
$$

since there are $m_{n_{1}}\left(V_{1}, U_{1}\right)$ choices for the first path, and $m_{n_{2}}\left(V-V_{1}, U-U_{1}\right)$ choices for the second path. The inequality follows from the fact that this operation is an injection into the set of paths of length $q p+n_{2}$ and with $V+q p-n_{1} B$-edge-visits and $U A$-edgevisits. Multiply this inequality by $w^{V} \alpha^{U}$ and sum over $V$ and over $U$. Since $\left|q p-n_{1}\right|<p$, this shows that

$$
Z_{n_{1}}(w, \alpha \mid p) Z_{n_{2}}(w, \alpha \mid p) \leq\left[\max \left\{1, w^{-p}\right\}\right] Z_{n_{1}+n_{2}+\left(q p-n_{1}\right)}(w, \alpha \mid p)
$$

This relation is enough to show that the limit in equation (2.2) exists ([31]).

In other words, to find the critical point in the complete model with generating function $G(z, w, \alpha \mid p)$, one only needs to consider a model of anchored Motzkin paths (see Theorem 5), and solve the equation $1+(1-\alpha) H(z, w, 1 \mid p)=0$. We cannot do this for general $w$, but in the case that $w=1$ we shall be able to find an asymptotic formula for the solution.

\subsection{Asymptotics for $H(1 / 3,1,1 \mid p)$ and $M(1 / 3,1,1 \mid p)$.}

In this section we find an asymptotic expression up to decaying terms for the location of the adsorption critical point in a Motzkin path model of $\left\{A B^{p-1}\right\}^{*}$ copolymer adsorption. Unfortunately, we have not been able to find this expression in its full generality, and instead we have been restricted to the case in which only $A$-interactions are considered i.e. when $v=1$ (for vertex colourings), and $w=1$ (for edge colourings). The proof will be based on the exchange relations in Theorems 2 and 5. In addition, an asymptotic expression for $M\left(z_{c}, 1,1 \mid p\right)$ will be needed, where $z_{c}=z_{c}(1,1 \mid p)$ is the radius of convergence of $M(z, 1,1 \mid p)$, and we pay attention first to this issue.

Let $m_{n}$ be the number of Motzkin paths of length $n$, and define the generating function

$$
M(z, 1)=M(z, 1,1 \mid 1)=\sum_{n=0}^{\infty} m_{n} z^{n}
$$

of Motzkin paths in a homopolymer model of polymer adsorption. In principle, $m_{n}$ can be computed from equation (21) using the contour integral

$$
m_{n}=\frac{1}{2 \pi i} \oint M(z, 1) \frac{\mathrm{d} z}{z^{n+1}} .
$$

There are established techniques for evaluating integrals of this type; for a general discussion of such techniques see, for example, [10]. In fact, the asymptotic evaluation of such 
integrals has been automated (see Bruno Salvy's gdev package ${ }^{3}$, which may be found at http://algo.inria.fr/salvy). For the above integral we summarise the application of these techniques. This is somewhat redundant but not lengthy and we do so to illustrate the ideas underlying the uniform asymptotic expansion of $m_{n}$.

This integral has two square root branch points at $z=-1$ and $z=1 / 3$, and by expanding the contour around the origin, one must only compute two integrals along branch cuts starting at these branch points. The contribution of the integral along the branch cut at $z=1 / 3$ grows as $3^{n}$, while the contribution of the integral along the branch cut at $z=-1$ has exponential behaviour $(-1)^{n}$, and both have sub-exponential dependence on $n$ otherwise. Asymptotically, this second contribution only gives a parity correction to $m_{n}$, it is dominated (in an exponential sense) by the first integral. Thus, after some simplification we obtain

$$
m_{n}=\frac{1}{2 \pi i} \int_{1 / 3}^{\infty} \frac{\sqrt{(1+z)(1-3 z)}}{z^{n+3}} \mathrm{~d} z+O\left((-1)^{n}\right) .
$$

Lemma 7. There exists an $M$ such that for sufficiently large $n$,

$$
\left|\frac{m_{n} 2 n \sqrt{n \pi}}{3^{n+3 / 2}}-\left(1-\frac{39}{16 n}+\frac{2665}{512 n^{2}}\right)\right|<\frac{M}{n^{3}} .
$$

Proof: We follow the techniques outlined in the proof of Theorem 5.2 in Chapter 5 of Flajolet and Sedgewick [10]. We must evaluate the integral in equation (35). Substitute $z=(1+s / n) / 3$, and expand the resulting integrand asymptotically (and uniformly) in $n$ to obtain

$$
\frac{3^{n+3} \sqrt{s}}{\pi e^{3 s} \sqrt{n^{3}}}\left(1+\frac{(72 s-69) s}{8 n}+\frac{\left(1296 s^{2}-6120 s+6471\right) s^{2}}{128 n^{2}}+O\left(n^{-3}\right)\right) .
$$

Using the arguments in Flajolet and Sedgewick, one may now integrate with respect to $s$ term by term. This completes the proof.

Asymptotics for $M(1 / 3,1,1 \mid p)$ can be obtained from lemma 7 . Since $H(1 / 3,1,1 \mid p)$ is closely related to $M(1 / 3,1,1 \mid p)$, its asymptotic expansion can also be derived.

Theorem 8. $M(1 / 3,1,1 \mid p)$ is asymptotic in $p$ to:

$$
M(1 / 3,1,1 \mid p) \sim 1+\frac{3 \sqrt{3}}{2 \sqrt{\pi p^{3}}}\left(\zeta(3 / 2)-\frac{39 \zeta(5 / 2)}{16 p}+\frac{2665 \zeta(7 / 2)}{512 p^{2}}+O\left(1 / p^{3}\right)\right)
$$

while the $H(1 / 3,1,1 \mid p)$ is asymptotic to:

$$
H(1 / 3,1,1 \mid p) \sim \frac{\sqrt{3}}{2 \sqrt{\pi p^{3}}}\left(\zeta(3 / 2)-\frac{15 \zeta(5 / 2)}{16 p}+\frac{505 \zeta(7 / 2)}{512 p^{2}}+O\left(1 / p^{3}\right)\right) .
$$

\footnotetext{
${ }^{3}$ We thank a referee for this reference.
} 
Proof: By lemma 7 there exists an $M>0$ such that for sufficiently large $p$ we have

$$
\left|m_{p} 3^{-p}-\frac{3 \sqrt{3}}{2 \sqrt{\pi}}\left(\frac{1}{p^{3 / 2}}-\frac{39}{16 p^{5 / 2}}+\frac{2665}{512 p^{7 / 2}}\right)\right| \leq \frac{M}{p^{9 / 2}} .
$$

Replace $p$ by $n p$ to find an asymptotic expression for the number of Motzkin paths of length $0 \bmod p$. Multiply this by $3^{-n p}$ and sum over $n \geq 1$. This shows that

$$
\left|\sum_{n=1}^{\infty} m_{n p} 3^{-n p}-\frac{3 \sqrt{3}}{2 \sqrt{\pi}}\left(\frac{\zeta(3 / 2)}{p^{3 / 2}}-\frac{39 \zeta(5 / 2)}{16 p^{5 / 2}}+\frac{2665 \zeta(7 / 2)}{512 p^{7 / 2}}\right)\right| \leq \frac{M \zeta(9 / 2)}{p^{9 / 2}} .
$$

Since $M(1 / 3,1,1 \mid p)=1+\sum_{n \geq 1} m_{n p} 3^{-n p}$ this gives the asymptotic expression for $M(1 / 3,1,1 \mid p)$. We find the asymptotic expression for $H(1 / 3,1,1 \mid p)=\sum_{n \geq 1} m_{n p-1} 3^{-n p}$ using similar reasoning to the above.

The expressions in this theorem can now be used to find an asymptotic expression for the adsorption critical points in the case that $w=1$.

Corollary 9. Consider the model of Motzkin paths with vertex-visits and generating function $M(z, v, a \mid p)$, and suppose that $v=1$. The critical point in this model is approximated asymptotically up to decaying terms by

$$
a_{c}(p)=\frac{2 \sqrt{3 \pi}}{9 \zeta(3 / 2)} p^{3 / 2}+\frac{13 \sqrt{3 \pi} \zeta(5 / 2)}{24 \zeta(3 / 2)^{2}} p^{1 / 2}+1+O\left(p^{-1 / 2}\right) .
$$

The Motzkin path model with edge-visits and generating function $M(z, w, \alpha \mid p)$, where $w=1$, has adsorption critical point approximated asymptotically in $p$ by

$$
\alpha_{c}(p) \sim \frac{2 \sqrt{3 \pi}}{3 \zeta(3 / 2)} p^{3 / 2}+\frac{5 \zeta(5 / 2) \sqrt{3 \pi}}{8 \zeta(3 / 2)^{2}} p^{1 / 2}+1+O\left(p^{-1 / 2}\right) .
$$

Proof. Let us first consider the vertex-visit case; the edge-visit case follows similarly. By examination of the structure of the generating function given in Theorem 3 we see that the singularities of $F(z, 1, a \mid p)$ are the singularities of $F(z, 1,1 \mid p), M(z, 1,1 \mid p)$ and the zero of the denominator. One can show that $F(z, 1,1 \mid p)$ has square root singularities at $z=\frac{1}{3},-1$, and that $M(z, 1,1 \mid p)$ has square-root singularities at $z=\frac{1}{3} \beta^{k}$ and $z=-\beta^{k}$, with $\beta$ a $p^{\text {th }}$ root of unity, $k=0,1, \ldots,(p-1)$. The zero of the denominator is given by

$$
M(z, 1,1 \mid p)=\frac{a}{a-1} \quad \text { or } \quad a=\frac{M(z, 1,1 \mid p)}{M(z, 1,1 \mid p)-1} .
$$

For small values of $a$, the dominant singularity is the square root singularity of $F$ and $M$ at $z=1 / 3$. For large values of $a$, the dominant singularity is the simple pole arising from the zero of the denominator. These two lines of singularities meet when $z=1 / 3$, and so there is a non-analytic point in the radius of convergence at

$$
a_{c}(p)=\frac{M(1 / 3,1,1 \mid p)}{M(1 / 3,1,1 \mid p)-1}
$$


which is interpreted as the location of the adsorption transition. Substituting the asymptotic expression for $M(1 / 3,1,1 \mid p)$ from Theorem 8 completes the proof.

It is interesting to note that

$$
\lim _{p \rightarrow \infty} \alpha_{c}(p) / a_{c}(p)=3
$$

and that asymptotically, $\alpha_{c}(p) \approx 3 a_{c}(p)$, but there seems to be no simple explanation for this. It is in principle also possible to consider other models of copolymer adsorption. In the case here it is possible to immediately extend the results to a model of $\left\{B A^{p-1}\right\}^{*}$ copolymer adsorption by suitable choices for $v$ and $a$ (or $w$ and $\alpha$ ). However, we cannot solve this case explicitly; that would require uniform (in $v$ or in $w$ ) asymptotics for $M(z, v, 1 \mid p)$ and $M(z, w, 1 \mid p)$ outside its radius of convergence, see [26].

\section{Adsorbing Bargraphs}

The techniques of Section 3 may be applied to other models, and in this section we consider a model of adsorbing bargraphs. There are also two possible models; the first a model of vertex-visit bargraphs, and the second a model of edge-visit bargraphs. The generating functions of these models can be obtained using standard techniques, at least in the homopolymer case. On the other hand, the copolymer cases are challenging, and we are only able to solve for the critical point in the case of a $\left\{A B^{p-1}\right\}^{*}$-copolymer with edge-visits: there are technical difficulties in the vertex-visit version of this model which makes the model (seemingly) intractable. We note that a number of periodic copolymer models have been solved for very short periods [22].

\subsection{The Vertex- and Edge-Adsorption Problem for Bargraphs}

As in the case of Motzkin paths, there is a canonical factorisation of bargraphs ${ }^{4}$ with edge-visits generated by $w$, and which can be implemented as follows:

\footnotetext{
${ }^{4}$ Note that the generating function $B(z, w)$ does not include the empty walk (a single vertex, having weight 1). This is deliberate; the inclusion of this term leads to a more complicated factorisation and more terms in the functional recurrence.
} 


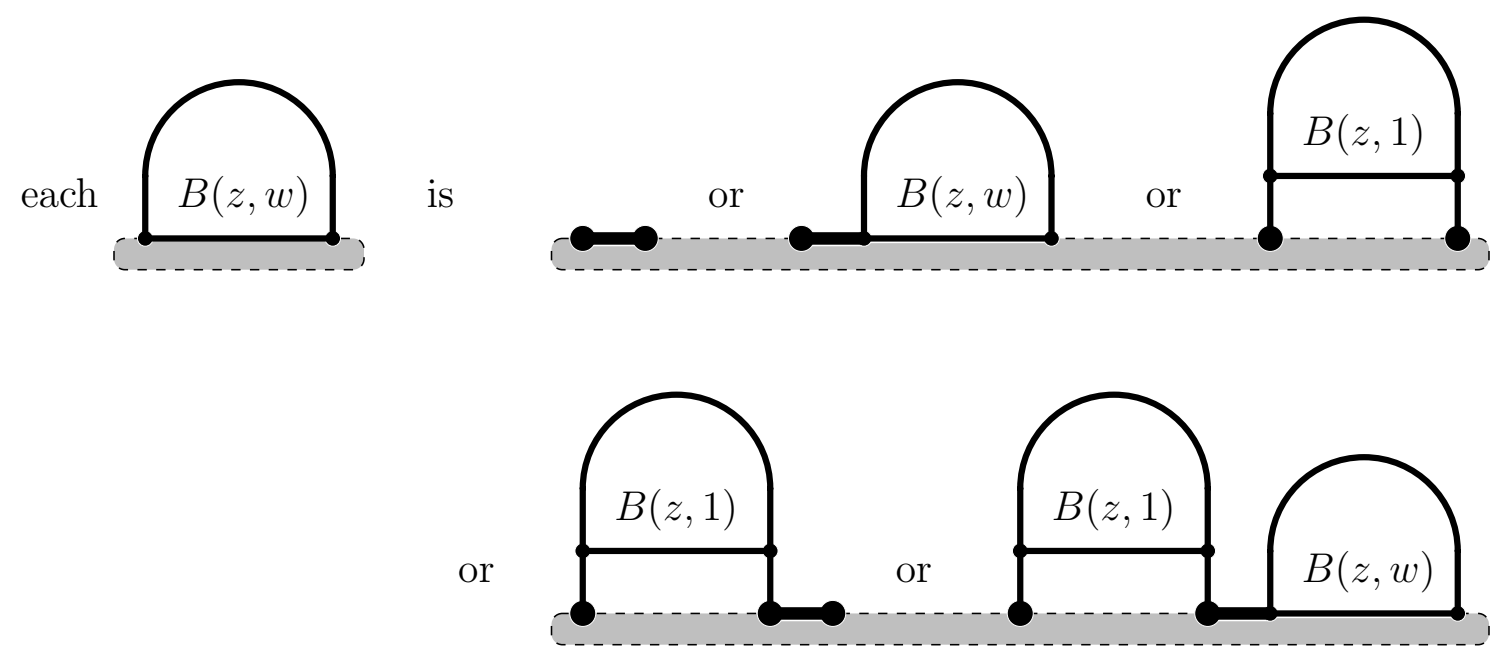

The factorization above leads to the following functional recurrence equation for $B(z, w)$ :

$$
B(z, w)=w z+z^{2} B(z, 1)+w z^{3} B(z, 1)+w z B(z, w)+w z^{3} B(z, 1) B(z, w) .
$$

This equation is solved by first finding a solution when $w=1$, which gives equation (8), and using this the full generating function can be written down from equation (45).

$$
B(z, w)=\frac{z\left(w+z B(z, 1)+z^{2} w B(z, 1)\right)}{1-w z-w z^{3} B(z, 1)} .
$$

The structure of this generating function is similar to that of Dyck paths; its radius of convergence is determined by a locus of square root singularities, and by a curve of simple poles when $1-w z-w z^{3} B(z, 1)=0$. The meeting of these is the adsorption critical point; for small values of $w$ the square root singularity determines the radius of convergence, while for large values of $w$ the curve of simple poles determine the radius of convergence. Consequently the asymptotic behaviour of $B(z, w)$ is similar to that of the Dyck path generating function $D(z, w)$ in that for small $w$ it is dominated by a square root singularity, while for large $w$ it is dominated by a simple pole, and the critical curve is a more complicated function of $w$ (it is the root of a degree six polynomial with coefficients that are polynomials in $w$ ). In spite of this, it is relatively simple to find the critical point by locating that value of $w$ where the square root singularity is replaced by the simple pole along the critical curve:

$$
w_{c}=\frac{2+\sqrt{2}}{2}, \quad \text { for bargraphs with edge-visits. }
$$

If a model of bargraphs with vertex-visits ${ }^{5}$ generated by $v$ is instead considered, then the recurrence equation is obtained from the factorisation in the figure above, and is explicitly given by

$$
B(z, v)=v^{2} z+v^{2} z^{2} B(z, 1)+v^{3} z^{3} B(z, 1)+v z B(z, v)+v^{2} z^{3} B(z, 1) B(z, v) .
$$

\footnotetext{
${ }^{5}$ The generating function $B(z, v)$ of these objects will not include the trivial walk consisting of one vertex and weight $v$; it can be added to $B(z, v)$ after the fact.
} 
Solving first for $B(z, 1)$ gives equation (8), but the generating function is in this case given by

$$
B(z, v)=\frac{v^{2} z\left(1+z B(z, 1)+v z^{2} B(z, 1)\right)}{1-v z-v^{2} z^{3} B(z, 1)}
$$

and the critical adsorption activity has numerical value

$$
v_{c}=(\sqrt{10}+\sqrt{5}-\sqrt{2}-1) / 2 .
$$

\subsection{Exchange Symmetry in Adsorbing Bargraphs with Edge- Visits}

A model of bargraph paths with edge-visits is similar to the model of Motzkin paths with edge-visits. The exchange relation in this model is identical to that of the corresponding Motzkin path model in equation (24), with appropriate reinterpretation of the generating functions. As before, it is necessary to define a model of anchored bargraphs, whose first edge lies horizontally in the adsorbing line.

Definition 3. Fix the period of the colouring $\left\{A B^{p-1}\right\}^{*}$ to be $p$. Define the following:

- $B(z, w, \alpha \mid p)$ is the generating function of bargraphs with edges labeled by $\left\{A B^{p-1}\right\}^{*}$ and of length $0 \bmod p$, edge-visits are generated by $w$, and wa generates A-edgevisits;

- $C(z, w, \alpha \mid p)$ is the generating function of all anchored bargraphs with edges labeled by $\left\{A B^{p-1}\right\}^{*}$ of length $0 \bmod p$ with all edge-visits generated by $w$, and A-visits generated by $w \alpha$.

An exchange relation satisfied by both $B(z, w, \alpha \mid p)$ and $C(z, w, \alpha \mid p)$ can be obtained using similar arguments to those leading to Theorem 5:

Theorem 10. The generating function $B(z, w, \alpha \mid p)$ satisfies the following exchange relation:

$$
\begin{aligned}
\alpha B(z, w, \alpha \mid p) C(z, w, 1 \mid p) & -(B(z, w, \alpha \mid p)-B(z, w, 0 \mid p)) \\
= & (B(z, w, \alpha \mid p)-B(z, w, 0 \mid p)) C(z, w, 1 \mid p)
\end{aligned}
$$

It follows that

$$
B(z, w, 1 \mid p)=B(z, w, 0 \mid p)(1+C(z, w, 1 \mid p))
$$

and so

$$
B(z, w, \alpha \mid p)=\frac{B(z, w, 1 \mid p)}{1+(1-\alpha) C(z, w, 1 \mid p)} .
$$


Proof. The proof of this theorem can be found by examining Figure 5. In the top figure a shaded and weighted (by $\alpha$ ) bargraph is followed by an unshaded and unweighted (by $\alpha$ ), anchored bargraph. The weighted path may be empty, but the unweighted path is non-empty. If the next $A$-edge-visit is weighted by an $\alpha$, then the bottom part of the figure is obtained, where the shaded and weighted path is non-empty, while the unshaded path may be empty. As for Motzkin and Dyck paths, the statistics of the top and bottom conformations only differ by one factor of $\alpha$, and the exchange relation follows.

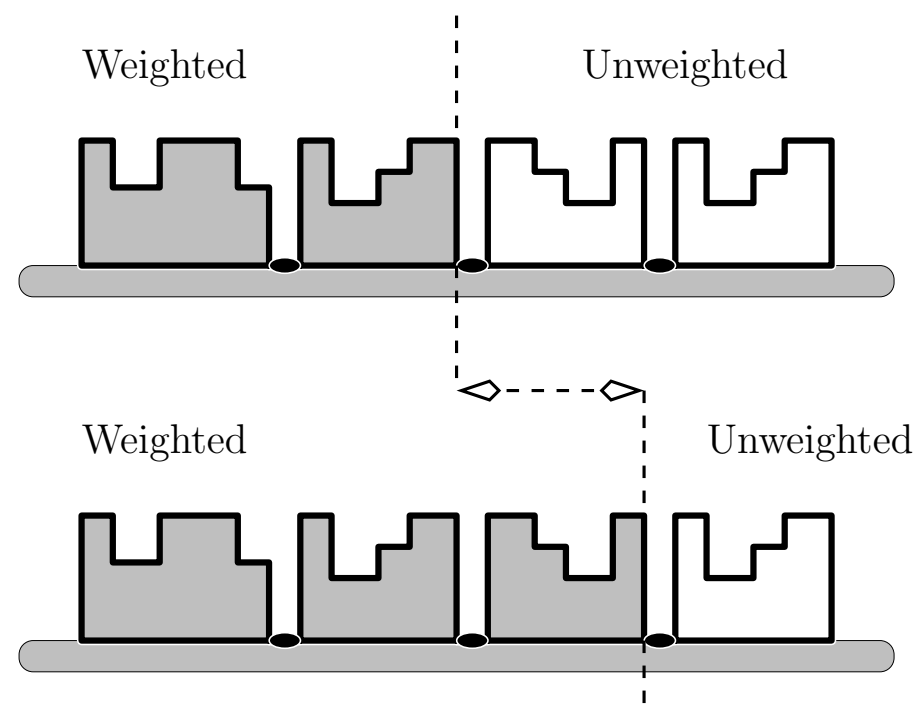

Figure 5: Edge-visit exchange relation.

The critical adsorption point is again located at the intersection of a curve of poles in $B(z, w, \alpha \mid p)$ in Theorem 10 given by

$$
1+(1-\alpha) C(z, w, 1 \mid p)=0
$$

and a line of branch points in $B(z, w, \alpha \mid p)$ along $z=\sqrt{2}-1$. Thus, the critical adsorption point in this model is located at $\alpha_{c}(p)$, which is the solution of

$$
1+(1-\alpha) C(\sqrt{2}-1, w, 1 \mid p)=0
$$

and we shall examine this below in the case that $w=1$.

\subsection{Vertex-coloured bargraphs}

In all the models we discussed previously, including vertex-coloured Dyck paths [26] and vertex-coloured Motzkin paths, the exchange relations express the fact that any coloured path ending in an $A$-visit may be concatenated with any unweighted path. Unfortunately this is not the case for the vertex-coloured bargraph model; in particular if the last edge in a bargraph is vertical, then it may not be concatenated with a bargraph whose first 
edge is vertical. This observation suggests that it will be challenging to find a simple exchange relation in this model; if we separate bargraphs into sets with last edge vertical or horizontal, then an exchange relation can be found, but it will involve two unknowns and cannot be solved to find either the generating function, nor the location of the critical adsorption point.

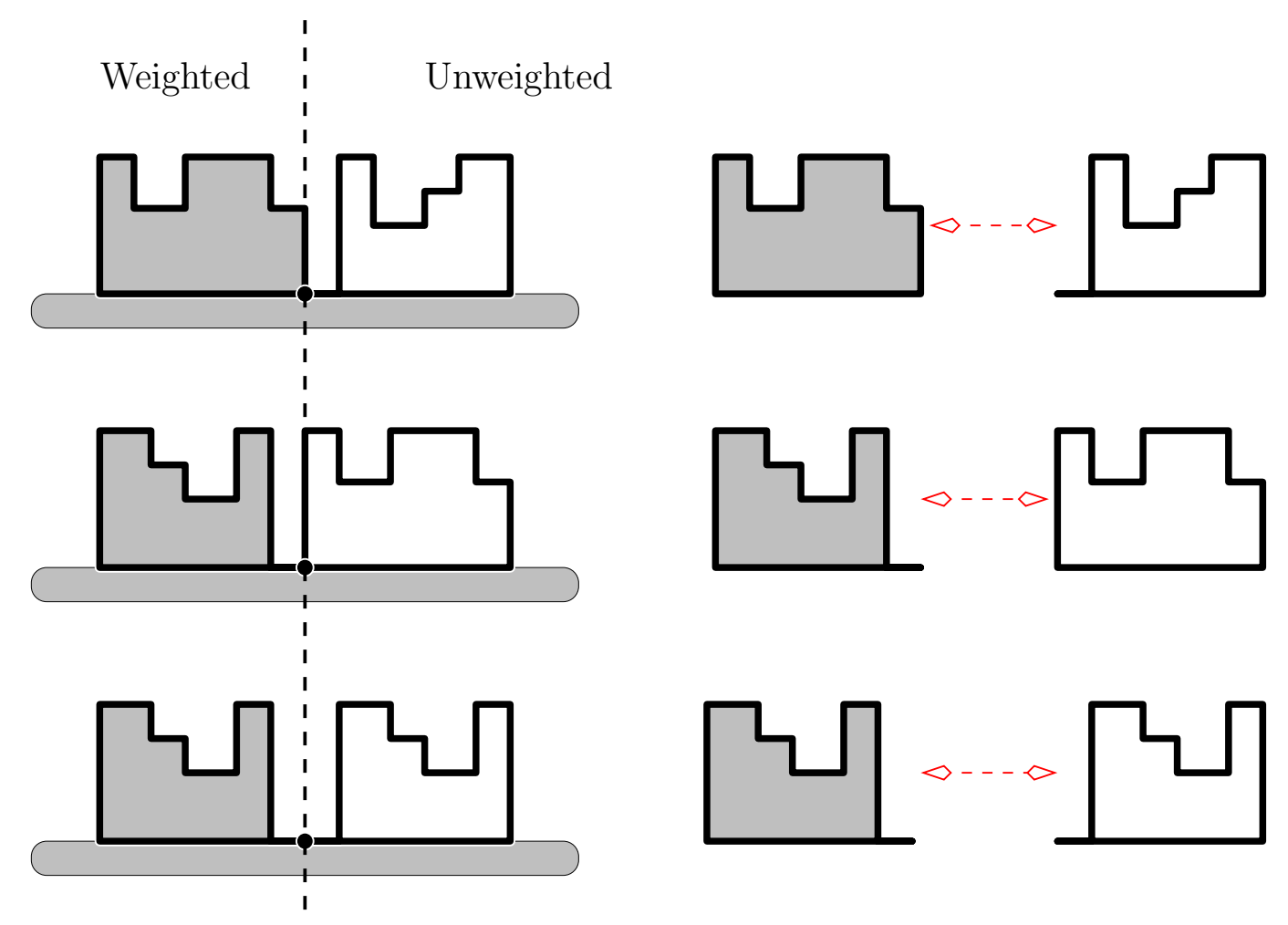

Figure 6: Permitted pairs of bargraphs. A bargraph that ends in a vertical edge may be joined to one that starts with a horizontal edge, but not a vertical edge. While a bargraph that ends in a horizontal step may be joined to any bargraph.

Definition 4. Define the following two generating functions of $\left\{A B^{p-1}\right\}^{*} A$ coloured bargraphs whose last edge is either vertical or horizontal (and where $z$ generates edges, $v$ generates vertex-visits (or either colour $A$ or $B$ ), and av generates A-vertex-visits.

- $X(z, v, a)$ is the generating function of vertex-coloured bargraphs that either consist of a single vertex or end in a horizontal edge and have length $0 \bmod p$.

- $Y(z, v, a \mid p)$ is the generating function of vertex-coloured bargraphs that end in a vertical edge and have length $0 \bmod p$.

Thus the generating function of all bargraphs is $B(z, v, a \mid p)=X(z, v, a \mid p)+Y(z, v, a \mid p)$, and the coefficient of $z^{0}$ in $X(z, v, a \mid p)$ is av. 
The following theorem follows by using similar reasoning to the proofs of other exchange relations.

Theorem 11. The generating functions $X(z, v, a \mid p)$ and $Y(z, v, a \mid p)$ satisfy the following exchange relation:

$$
\begin{aligned}
a(X(a)(X(1)-v)+X(a) Y(1)+Y(a)(X(1)-v)) & = \\
(X(a)-v a) X(1)+(X(a)-v a) Y(1) & +Y(a) X(1)
\end{aligned}
$$

where we have used $X(a)$ and $Y(a)$ as shorthand for $X(z, v, a \mid p)$ and $Y(z, v, a \mid p)$.

Unfortunately this exchange relation involves two unknowns, namely $X(z, v, a \mid p)$ and $Y(z, v, a \mid p)$, and so may not be solved, nor have we been able to extract the location of the critical adsorption point from it.

\subsection{Asymptotics of $C(\sqrt{2}-1,1,1 \mid p)$.}

In this section we develop asymptotics of $C(\sqrt{2}-1,1,1 \mid p)$ which can be used to find an asymptotic expression for the location of the adsorption critical point in $\left\{A B^{p-1}\right\}^{*}$-edgecoloured copolymers. In this model we only consider the case that $w=1$, and we find the asymptotics for $\alpha_{c}(p)$ only as a function of $p$.

Let $b_{n}$ be the number of bargraphs of length $n$. Then

$$
B(z, 1,1 \mid p)=\sum_{n=0}^{\infty} b_{n p} z^{n p}
$$

Anchored bargraphs are counted by $b_{n p-1}$, and by adding one extra factor of $z$ to generate the first horizontal edge, one obtains

$$
C(z, 1,1 \mid p)=\sum_{n=0}^{\infty} b_{n p-1} z^{n p}
$$

Hence, only the asymptotics of $b_{n}$ are needed in order to find the asymptotics of $C(z, 1,1 \mid p)$. The radii of convergence of $B(z, 1,1 \mid p)$ and $C(z, 1,1 \mid p)$ are both given by $z_{c}=\sqrt{2}-1$; this defines part of the critical curve of $C(z, 1, a \mid p)$ in Theorem 10.

Lemma 12. There exists a finite constant $M$, such that for sufficiently large $n_{0}>0$, and for every $n \geq n_{0}$,

$$
\left|b_{n} \sqrt{\pi n^{3}}(\sqrt{2}-1)^{n+3 / 2}-\left(1-\frac{21 \sqrt{2}+36}{16 n}+\frac{5(252 \sqrt{2}+349)}{256 n^{2}}\right)\right|<\frac{M}{n^{3}} .
$$

Proof. The proof of the above is similar to that of Lemma 7. 
Theorem 13. The function $C(\sqrt{2}-1,1,1 \mid p)$ is asymptotic to

$$
\begin{aligned}
C(\sqrt{2}-1,1,1 \mid p) & \sim \sqrt{\frac{1+\sqrt{2}}{\pi}} \times \\
& \left(\frac{\zeta(3 / 2)}{p^{3 / 2}}-\frac{(12+21 \sqrt{2}) \zeta(5 / 2)}{16 p^{5 / 2}}+\frac{5(157+357 \sqrt{2}) \zeta(7 / 2)}{256 p^{7 / 2}}+O\left(p^{-9 / 2}\right)\right) .
\end{aligned}
$$

Consider first equation (58). Replace $b_{n p-1}$ by its asymptotic expression from lemma 12 , and expand it asymptotically in $p$. If $z=\sqrt{2}-1$, then the sum over $n$ can be done termby-term. This proves the theorem.

An immediate consequence of this theorem is an asymptotic formula for $\alpha_{c}(p)$.

Corollary 14. The critical adsorption point for adsorbing bargraphs with edge-visits has the following asymptotic expression:

$$
\alpha_{c}(p)=\frac{2 \sqrt{\pi} \sqrt{\sqrt{2}-1}}{\zeta(3 / 2)} p^{3 / 2}+\frac{3 \zeta(5 / 2) \sqrt{\pi} \sqrt{58 \sqrt{2}-2}}{\zeta(3 / 2)^{2}} p^{1 / 2}+1+O\left(p^{-1 / 2}\right) .
$$

Proof. The critical point is given by the solution of equation (55); substitute the asymptotic formula for $C(\sqrt{2}-1,1,1 \mid p)$ from Theorem 13 and solve for $a$.

\section{Acknowledgements}

The authors would like to thank C. Chauve, M. Zabrocki, M. Bousquet-Mélou and S. G. Whittington for their helpful discussions. E. J. Janse van Rensburg is supported by an operating grant from NSERC (Canada).

\section{References}

[1] C. Banderier and P. Flajolet, 2001, Basic Analytic Combinatorics of Directed Lattice Paths. To appear in Theo. Comp. Sci.

[2] R. Brak, A.L. Owczarek and T. Prellberg, 1993, A Scaling Theorey of the Collapse Transition in Geometric Cluster Models of Polymers and Vesicles. J. Phys. A: Math. Gen. 26, 4565-4579.

[3] M. Bousquet-Mélou, 1996, A Method for the Enumeration of Various Classes of Column-Convex Polygons, Discrete Math., 154, 1-25.

[4] R. Brak, A.J. Guttmann and S.G. Whittington, 1991, On the Behaviour of Collapsing Linear and Branched Polymers, J. Math. Chem. 8, 255-68.

[5] R. Brak, A.J. Guttmann and S.G. Whittington, 1992, A Collapse Transition in a Directed Walk Model, J. Phys. A: Math Gen. 25, 2437-46. 
[6] R. Brak, J.M. Essam and A.L. Owczarek, 1998, New Results for Directed Vesicles and Chains near an Attractive Wall. J. Stat. Phys. 93, 155-192.

[7] A. R. Conway and A. J. Guttmann, 1996, Square Lattice Self-Avoiding Walks and Corrections to Scaling. Phys. Rev. Lett., 77, 5284-5287.

[8] K. De'Bell and T. Lookman, 1993, Surface Phase Transitions in Polymer Systems. Rev. Mod. Phys. 65, 87-114.

[9] M.-P. Delest and X.G. Viennot, 1984, Algebraic Languages and Polyominoe Enumeration. Theo. Comp. Sci. 34, 169-206.

[10] P. Flajolet and R. Sedgewick, 2001, Analytic Combinatorics: Singularity Analysis of Generating Functions. INRIA Rapport de Recherche No. 4103, January 2001, chapter 5 .

[11] I.M. Gessel, 1986, A Probabilistic Method for Lattice Path Enumeration. J. Stat. Plan. and Infer. 14, 49-58.

[12] A. J. Guttmann, I. Jensen, L. H. Wong and I. G. Enting, 2000, Punctured Polygons and Polyominoes on the Square Lattice. J. Phys. A: Math. Gen. 33, 1735-1764.

[13] J.M. Hammersley, G.M. Torrie and S.G. Whittington, 1982, Self-Avoiding Walks Interacting with a Surface. J. Phys. A: Math. Gen. 15, 539-571.

[14] E.J. Janse van Rensburg, 1998, Collapsing and Adsorbing Polygons. J. Phys. A: Math. Gen. 31, 8295-8306.

[15] E.J. Janse van Rensburg, 1999, Adsorbing Staircase Walks and Staircase Polygons. Ann. Comb. 3, 451-473.

[16] E.J. Janse van Rensburg, 2000, The Statistical Mechanics of Interacting Walks, Polygons, Animals and Vesicles. Oxford Lecture Series in Mathematics and its Applications 18 (OUP Inc., New York).

[17] P. Larcombe and P. D. C. Wilson, 1998, On the trail of the Catalan sequence. Mathematics Today $\mathbf{3 4}, 114-117$.

[18] P. Larcombe, 1999, On the history of the Catalan numbers: a first record in China, Mathematics Today 35, 89.

[19] I.D. Lawrie and S. Sarlbach, 1984, Tricriticality. In Phase Transitions and Critical Phenomena 9, 65-161. Eds.: C. Domb and J.L. Lebowitz (Academic Press, London).

[20] J. J. Luo, 1993, Catalan numbers in the history of mathematics in China. In Combinatorics and graph theory: proceedings of the spring school and international conference on combinatorics, Hefei, 6-27 April 1992, 68-70. Eds.: H. P. Yap, T. H. Ku, E. K. Lloyd and Z. M. Wang (World Scientific). 
[21] N. Madras and G. Slade, 1993, The Self-Avoiding Walk. Probability and its Applications (Birkhäuser, Boston).

[22] M.S. Moghaddam, T. Vrbova and S.G. Whittington, 2000, Adsorption of periodic copolymers at a planar interface, J. Phys. A: Math. Gen. 33, 4573-4584

[23] A.L. Owczarek, T. Prellberg and R. Brak, 1993, New Scaling Form for the Collapsed Polymer Phase. Phys. Rev. Lett. 70, 951-953; - 1993, The Tricritical Behaviour of Self-Interacting Partially Directed Walks. J. Stat. Phys. 72, 737-772.

[24] T. Prellberg and R. Brak, 1995, Critical Exponents from Nonlinear Functional Equations for Partially Directed Cluster Models. J. Stat. Phys. 78, 701-730.

[25] V. Privman, G. Forgacs and H.L. Frisch, 1988, New Solvable Models of Polymer Chain Adsorption near a Surface. Phys. Rev. B37, 9897-9900.

[26] A. Rechnitzer and E.J. Janse van Rensburg, 2001, Exchange Relations, Dyck Paths and Copolymer Adsorption. Submitted to Applied Discrete Math.

[27] R. Stanley, 1999, Enumerative Combinatorics Volume II. Cambridge University Press.

[28] H.N.V. Temperley, 1956, Combinatorial Problems suggested by the Statistical Mechanics of Domains and of Rubber-like Molecules. Phys. Rev. 103, 1-16.

[29] T. Vrbová and S.G. Whittington, 1996, Adsorption and Collapse of Self-Avoiding Walks and Polygons in Three Dimensions. J. Phys. A: Math. Gen. 29, 6253-6264;1998, Adsorption and Collapse of Self-Avoiding Walks in Three Dimensions. J. Phys. A: Math. Gen. 31, 3989-3998; - 1998, Adsorption and Collapse of of Self-Avoiding Walks at a Defect Plane. J. Phys. A: Math. Gen. 31, 7031-7041.

[30] S.G. Whittington, 1998, A Directed Walk Model of Copolymer Adsorption. J. Phys. A: Math. Gen. 31, 8797-8803.

[31] J.B. Wilker and S.G. Whittington, 1979, Extension of a Theorem on SuperMultiplicative Functions. J. Phys. A: Math. Gen. 12, L245-L247. 